\title{
Contents
}

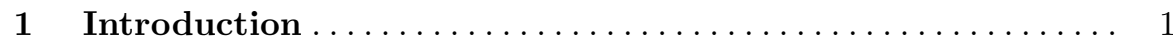

\section{Part I State of the Art}

2 Basic Equations and Dimensionless Numbers $\ldots \ldots \ldots \ldots .7$

2.1 Dimensionless Numbers and Time Scales .............. 8

2.1 .1 Global Time Domain ........................ 9

2.1 .2 Initial Time Domain ........................ 10

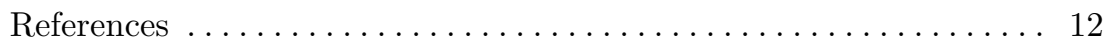

3 Orders of Magnitude of the Dimensionless Numbers ...... 13

References .................................. 15

4 Literature Review ............................ 17

4.1 Liquid Management in Space ...................... 17

4.2 Surface Tension Tank Technology ..................... 18

4.3 Contact Angle - Isothermal Conditions . . . . . . . . . . . . . 19

4.3.1 Dynamic Contact Angle..................... 23

4.3.2 Oscillating Contact Line ................... 30

4.4 Contact Angle - Non-isothermal Conditions ............. 32

4.5 Surface Oscillations . . . . . . . . . . . . . . . . . . . 34

4.6 Capillary Rise in Tubes and Vanes.................. 36

4.7 Critical Velocities in Open Capillary Channel Flows ........ 42

References ................................... 44

\section{Part II Surface Oscillations upon Step Reduction} of the Bond Number

$5 \quad$ Static Surface Shapes .......................... 51

5.1 Initial Condition with Residual Acceleration .............. 51 
5.2 Surface Shape in Reduced Gravity . . . . . . . . . . . . . . . . 53

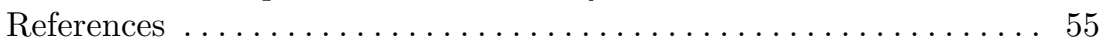

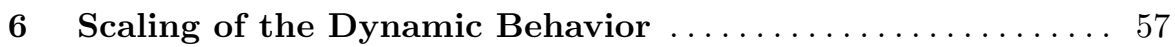

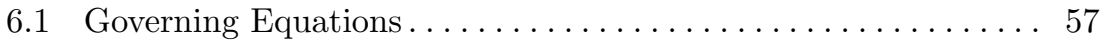

6.2 Scaling of the Initial Capillary Rise (Initial Time Domain) . . . 58

6.3 Scaling of the Surface Oscillation (Global Time Domain) . . . 60

References .......................... 62

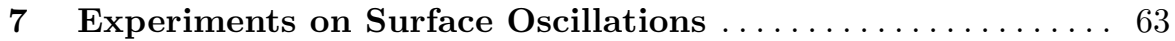

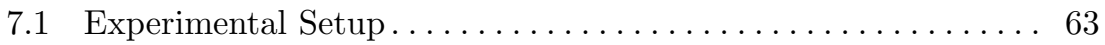

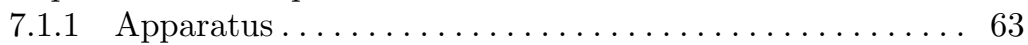

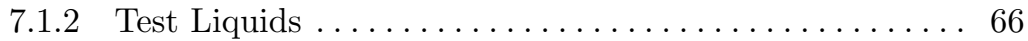

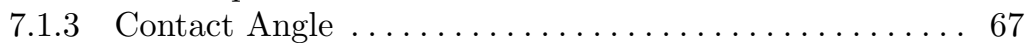

7.1 .4 Test Parameters........................ 67

7.2 Data Evaluation. . . . . . . . . . . . . . . . . . . 72

7.2.1 Definition of the Experimental Time ........... 73

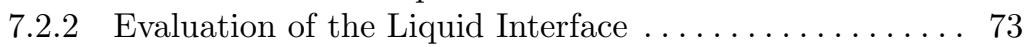

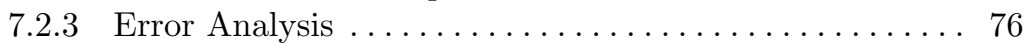

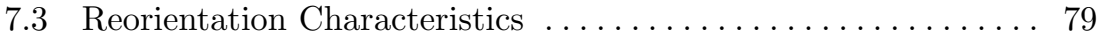

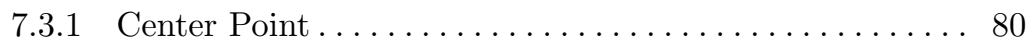

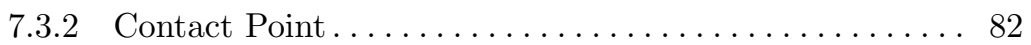

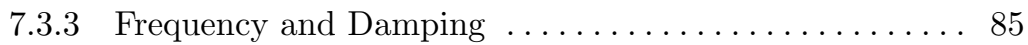

References ................................. 90

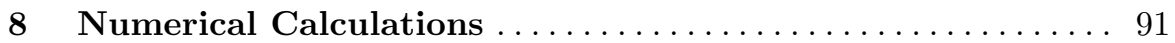

8.1 Numerical Model used in FIDAP . . . . . . . . . . . . . . . . 91

8.2 Numerical Model used in FLOW-3D . . . . . . . . . . . . . . 94

8.3 Data Evaluation . . . . . . . . . . . . . . . . . . . . 96

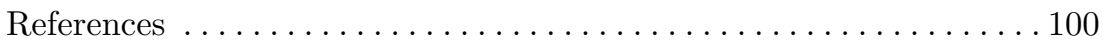

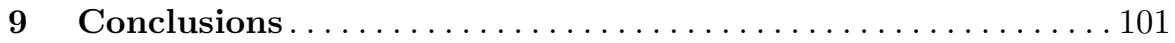

9.1 Contact Point Observations . . . . . . . . . . . . . . . . . 101

9.1.1 Experimental Observations for the Contact Point . . . . . 101

9.1.2 Numerical Observation for the Contact Point . . . . . . 107

9.2 Summary Contact Point Observations . . . . . . . . . . . . . . 112

9.3 Center Point Observations . . . . . . . . . . . . . . . . . 115

9.3.1 Experimental Observations for the Center Point . . . . . 115

9.3.2 Numerical Observations for the Center Point. . . . . . . . 122

9.4 Summary Center Point Observations . . . . . . . . . . . . . . . . . . . 124

9.4.1 Frequency of Oscillation . . . . . . . . . . . . . . . 124

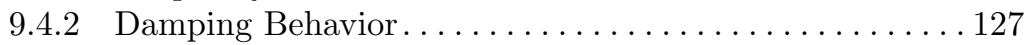

9.4.3 First Amplitude . . . . . . . . . . . . . . . . . . 129

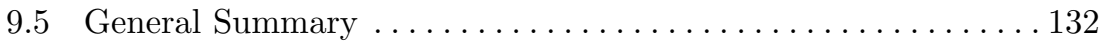

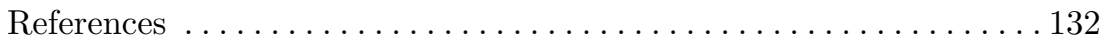


Part III Capillary Rise in Cylindrical Tubes

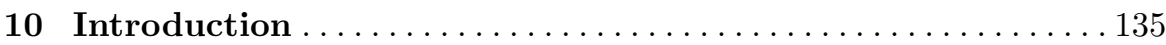

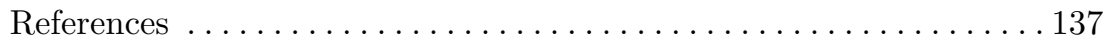

11 Experiments on Capillary Rise. . . . . . . . . . . . . . . . . 139

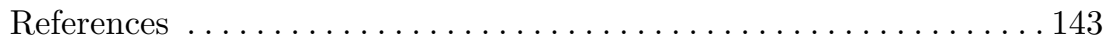

12 Mathematical Model for the Capillary Rise . . . . . . . . . . . 145

12.1 Pressure Force at the Upper Boundary . . . . . . . . . . . . . . 147

12.2 Pressure Force at the Lower Boundary . . . . . . . . . . . . . . . . 148

12.3 Friction Force inside the Tube . . . . . . . . . . . . . . . . . . . . . . 149

12.4 Equation for the Capillary Rise and Variation

of the Coefficients ......................... 151

12.5 Dimensionless Equation for the Capillary Rise. . . . . . . . . . 152

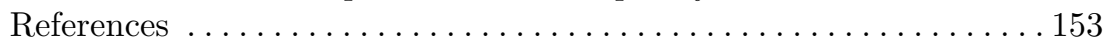

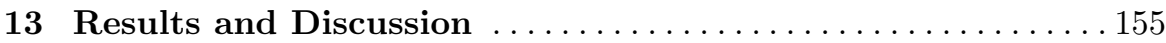

13.1 Comparison of Experimental and Theoretical Results . . . . . . 155

13.2 Dimensionless Representation of the Results . . . . . . . . . . 156

13.3 Comparison of the Dominating Forces . . . . . . . . . . . . 165

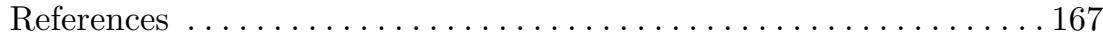

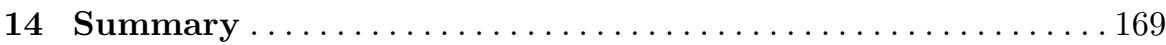

\section{Part IV Critical Velocities in Open Capillary Channel Flows}

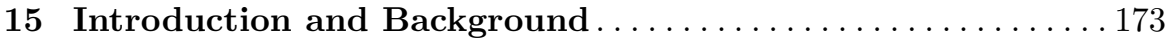

15.1 Application for Liquid Management in Space......... . . . . 175

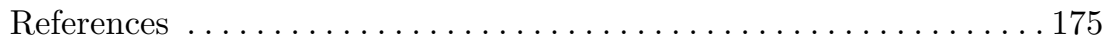

16 Mathematical Model for the Flow Between Parallel Plates . 177

16.1 Pressure Term and Curvature . . . . . . . . . . . . . . . . 178

16.2 Convective Momentum Transport . . . . . . . . . . . . . . . . . 180

16.3 Molecular Momentum Transport . . . . . . . . . . . . . . . 180

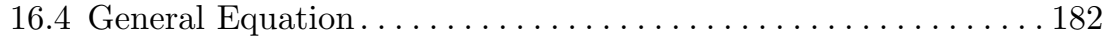

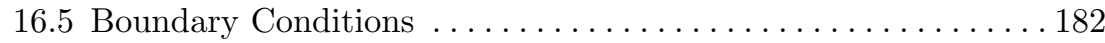

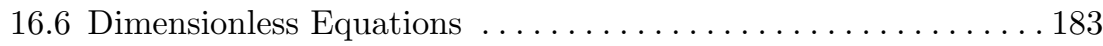

16.7 Numerical Solution . . . . . . . . . . . . . . . . . . . . . . . . . . . . . . . . . . . 185

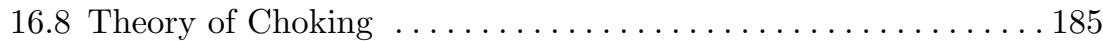

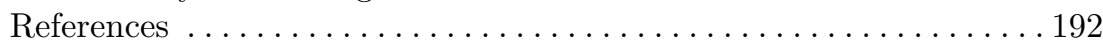


17 Experimental Results . . . . . . . . . . . . . . . . . . . 193

17.1 Experiments in a Drop Tower . . . . . . . . . . . . . . . . . . 194

17.2 Experiment on a Sounding Rocket ... . . . . . . . . . . 200

17.2.1 Filling of the Compensation Tube ............ 207

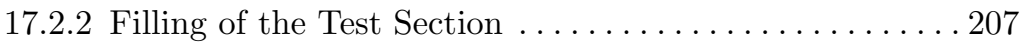

17.2.3 Coarse Approach to the Critical Velocity . . . . . . . . . . 208

17.2.4 Fine Approach to the Critical Velocity . . . . . . . . . . . 209

17.2.5 Data Evaluation Compensation Tube . . . . . . . . . . . 209

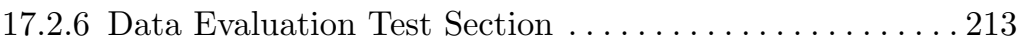

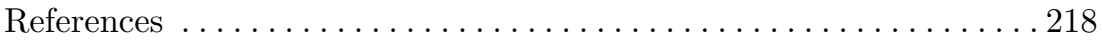

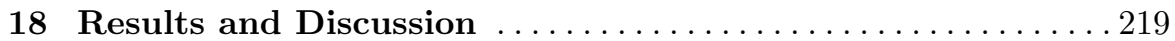

\section{Part V Perspectives}

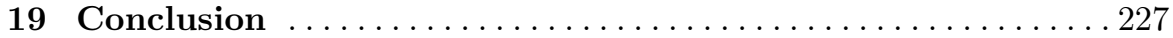

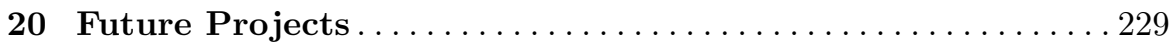

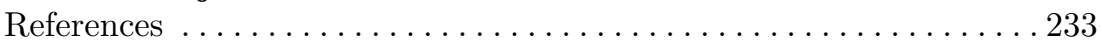

\section{Part VI Appendix}

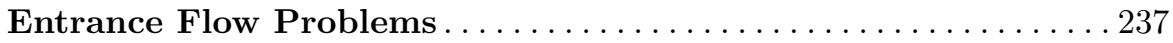

A.1 Analytical Calculation of the Inlet Pressure Drop . . . . . . . . . . 237

A.2 Numerical Calculation of the Inlet Pressure Drop . . . . . . . . . . 243

A.2.1 Computation of the Inlet Pressure Loss into a Cylindrical Tube from an Infinite Reservoir . . . . . . . . 243

A.2.2 Computation of the Inlet Pressure Loss into a Rectangular Channel from an Infinite Reservoir through a Nozzle . . . . . . . . . . . . . . . . 249

A.3 Determination of the Entrance Pressure Distribution . . . . . . . 251

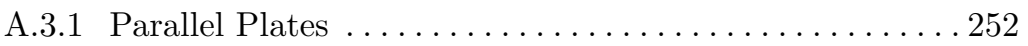

A.3.2 Circular Cylindrical Tube . . . . . . . . . . . . . . 259

A.3.3 Numerical Calculation of the Entrance Pressure Drop . . 260

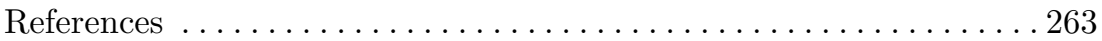

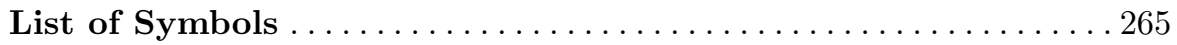

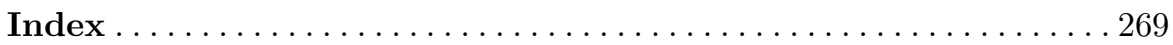

